\title{
Nano-lantern lights the way
}

The bright luminescent protein Nanolantern is easily modified to create functional sensors.

Though the rainbow of fluorescent proteins now available has transformed cell biology, fluorescence imaging cannot be used in all situations. Fluorescence excitation requires an external light source, which makes this technique ill suited for deep tissue imaging (owing to light scattering), prolonged observations (owing to photobleaching and phototoxicity) or applications in which the substrate of interest is autofluorescent (owing to image interference). Fluorescence imaging is also not very compatible in combination with approaches that also require a light-based trigger, such as optogenetics.

With luminescence, however, light is generated by a localized chemical reaction, without the need for external illumination. But the light given off by available luminescent proteins, even those based on a bioluminescence resonance energy transfer (BRET) mechanism, is quite dim. Thus, despite its advantages, luminescence imaging has remained underexploited.

Takeharu Nagai of Osaka University and Hokkaido University and his colleagues now offer up a much brighter luminescent protein called Nano-lantern. This probe consists of an enhanced Renilla luciferase (RLuc) variant fused to the yellow fluorescent protein Venus. Venus serves as a BRET acceptor to RLuc's luminescent substrate coelenterazine to boost brightness. When expressed in HeLa cells, Nano-lantern allowed the researchers to record luminescence images that were nearly comparable to fluorescence images, even allowing the imaging of fine features such as microfilaments and microtubules. Nano-lantern also allowed imaging of small subcutaneous tumors at video rates in freely moving mice.
The RLuc moiety of Nano-lantern contains an unstructured loop region into which functional sensor domains can be inserted. Nagai's team created luminescent sensors to observe $\mathrm{Ca}^{2+}$, cAMP and ATP dynamics in living cells. This allowed them to image situations in which it is not possible to use fluorescence, such as in combined $\mathrm{Ca}^{2+}$ imaging with optogenetic control in neurons or in the detection of ATP in strongly autofluorescent plant chloroplasts.

Still, Nano-lantern's design has room for improvement. The RLuc substrate is rapidly consumed, making it challenging to continuously observe biological events. Further increases in brightness will also expand applicability.

\section{Allison Doerr}

\section{RESEARCH PAPERS}

Saito, K. et al. Luminescent proteins for high-speed single-cell and whole-body imaging. Nat. Commun. 3, 1262 (2012). 